\title{
Thermodynamic state of the interface during acoustic cavitation in lipid suspensions
}

\author{
Shamit Shrivastava* and Robin O. Cleveland \\ Department of Engineering Science, University of Oxford, Oxford, OX1 3PJ, United Kingdom
}

(Received 9 August 2018; revised manuscript received 26 January 2019; published 9 May 2019)

\begin{abstract}
The thermodynamic state of lipid interfaces was observed during shock wave induced cavitation in water with submicrosecond resolution, using the emission spectra of hydration-sensitive fluorescent probes colocalized at the interface. The experiments show that the cavitation threshold is lowest near a phase transition of the lipid interface. The cavitation collapse time and the maximum state change during cavitation are found to be a function of both the driving pressure and the initial state of the lipid interface. The experiments show dehydration and crystallization of lipids during the expansion phase of cavitation, suggesting that the heaporization is absorbed from within the interface, which is adiabatically uncoupled from the free water. The study underlines the critical role of the thermodynamic state of the interface in cavitation dynamics, which has mechanistic implications for ultrasound-mediated drug delivery, acoustic nerve stimulation, ultrasound contrast agents, and the nucleation of ice during cavitation.
\end{abstract}

DOI: 10.1103/PhysRevMaterials.3.055602

\section{INTRODUCTION}

Cavitation is the formation of vapor cavities in a liquid due to a tensile stress [1] and is assumed to be one of the key mechanisms for biophysical effects of ultrasound and in particular shock waves [2,3]. It has been suggested as a possible mechanism both in transmembrane and intracellular drug delivery [4] as well as acoustic excitation of the peripheral nervous system [5]. The key biophysical system in both these cases is the cellular membrane, which is essentially a self-assembled macromolecular bilayer mainly composed of lipids. To determine the dynamics of the membrane during cavitation it is necessary to monitor the membrane with microsecond temporal resolution. Direct high-speed imaging provides morphological images of cell deformation [6] but not the thermodynamic state changes that can be achieved through fluorescence dyes [7]. Fluorescent dyes have played a fundamental role in explaining native dynamic processes in biological membranes, such as membrane structural changes during a nerve impulse [8]. Here a fluorescent dye is employed to probe the dynamics of aqueous lipid membranes resulting from cavitation.

Lipid surfactants provide a way of controlling the thermodynamic state of an interface and have been investigated in great detail, partly because of their ubiquity in biological systems $[9,10]$. Thermodynamically, lipid membranes can be treated as quasi-two-dimensional (2D) systems, where the state can be defined by surface observables (e.g., area/molecule) and corresponding thermodynamic fields (e.g., surface pressure) [11]. Molecules which colocalize at the interface, such as fluorescent probes, can be added to the system and used to indicate the thermodynamic state of the interface. For example, we recently showed that the wavelength shift of the emission spectrum of the fluorescent probe Laurdan can be used to dynamically measure the thermodynamic state of lipid

\footnotetext{
*shamit.shrivastava@eng.ox.ac.uk
}

interfaces [7], when subjected to microseconds-width acoustic impulses.

The enthalpy of the vapor-liquid interface during cavitation can be expressed as $\Delta H_{i}=T_{i} \Delta S_{i}+A \Delta \sigma$, where $\sigma$ is the surface tension, $A$ is the surface area, $T_{i}$ is the interfacial temperature, and $S_{i}$ is the interfacial entropy [12,13]. At the formation of a cavity in water under normal pressure and temperature, $\Delta \sigma$ is typically of the order of $72 \mathrm{mN} / \mathrm{m}$, giving a theoretical tensile strength or the cavitation threshold of water in excess of $100 \mathrm{MPa}$ [14]. However, the tensile strength of tap water is practically of the order of $0.1 \mathrm{MPa}$ [15] which can be attributed to the presence of particles with cracks and crevices that stabilize cavitation nuclei [16] or to impurities acting as surfactants that lower the surface tension. The latter case is relevant to this work, as the cavitation threshold has been shown to be significantly lower for fat-water or oilwater interfaces than in either pure water or pure oil [17], and cavitation has been observed to initiate specifically at the interfaces [1]. Subsequent macroscopic expansion of the cavity in the presence of surfactants has been studied widely in the context of lipid-coated bubbles; see, for example, the review by Doinikov and Bouakaz [18]. Thus the surfactants can affect not only the nucleation but also the subsequent macroscopic evolution, which several models have tried to capture by allowing for a radius-dependent surface tension, $\sigma(R)$ [19] in the Rayleigh-Plesset equations, and making the assumption that the energy of the interface is given by $\Delta E_{i}=$ $\sigma \Delta A$. However, this ignores the entropy or the heat content of the interface. The complete expression is $\Delta E_{i}=\sigma \Delta A+$ $T_{i} \Delta S_{i}$ [13], where $\Delta S_{i}=-\frac{d \sigma(T)}{d T} \Delta A$ [13] and this study will show that the entropy term is significant and is required to explain the observed role of the interface during cavitation, even qualitatively.

Here we investigate cavitation in a suspension of lipid vesicles by observing the spectral shift in the fluorescence emission of a hydrophobic probe, Laurdan, colocalized at the interface [7] with submicrosecond resolution. The lipid 
composition and the temperature of the suspension were varied to change the initial state of the lipid interfaces. The cavitation was generated by firing focused acoustic shock waves into the aqueous suspension. No a priori assumptions about the site or the spatial details of the evolving cavitation field were made; rather they were deduced from the experimental observations presented in this study.

\section{MATERIAL AND METHODS}

The experimental setup and the multilamellar lipid vesicle (MLV) preparation have been described in detail previously [7]. The lipids 1,2-dioleoyl-sn-glycero-3phosphocholine (DOPC 850375), 1,2-dimyristoyl-sn-glycero3-phosphocholine (DMPC 850345), and 1,2-dipalmitoyl-snglycero-3-phosphocholine (DPPC 850355) were purchased as a $25 \mathrm{mg} / \mathrm{ml}$ solution in chloroform from Avanti Polar Lipids, Inc. (Alabaster, AL, USA). The flourescent probe Laurdan was purchased from Thermo Fisher Scientific (Waltham, MA, USA). MLVs were prepared from $2.5 \mathrm{mg}$ of one of the lipids (DOPC, DMPC, or DPPC) and $50 \mu \mathrm{g}$ of Laurdan and added to a temperature controlled water tank containing $500 \mathrm{ml}$ of deionized water, equilibrated to the experimental temperature. For determining the cavitation threshold $12.5 \mathrm{mg}$ of lipids were extruded through a $100-\mathrm{nm}$ polycarbonate filter at $60^{\circ} \mathrm{C}$ and added to $500 \mathrm{ml}$ of de-ionized ( $18 \mathrm{M} \Omega$ resistance) and degassed water. The lipid nanoparticles were sized using differential light scattering (Malvern Panalytical, Zeta Sizer, Spectris USA). At $24^{\circ} \mathrm{C}$ and $p \mathrm{H} 7$, the mean hydrodynamic radius and polydispersity index were as follows: DOPC $(160 \mathrm{~nm}$, $0.2)$, DMPC (140 nm, 0.17), and DPPC (452 nm, 0.44).

The phase state of each lipid was characterized in terms of a "reduced temperature," $T^{*}=\frac{T-T_{m}}{T_{m}}$, where $T_{m}$ is the temperature of the main phase transition, equivalent to the melting point of of the lipid system. Therefore when $T^{*}<0$ the lipid is in the $L_{\beta}$ gel phase, when $T^{*}>0$ it is in the $L_{\alpha}$ fluid phase, and when $T^{*}=0$ it is at transition.

Shock waves generated by a Swiss PiezoClast ${ }^{\circledR}$ (EMS Electro Medical Systems S.A., Switzerland) were fired into a small test chamber that had been filled with the lipid solution, and focused $1 \mathrm{~cm}$ below the free surface [Fig. 1(a)]. The pressure waveforms generated by the PiezoClast were measured with a PVDF needle hydrophone (Müller-Platte needle probe, Dr. Müller instruments, Oberursel, Germany) with a manufacturer specified sensitivity of $12.5 \mathrm{mV} / \mathrm{MPa}$. The needle was placed at the focus of the PiezoClast, i.e., $1 \mathrm{~cm}$ below the free surface, and measured shock waves at four different energy levels are shown in Fig. 1(b). The waveforms exhibit a leading positive pressure followed by a negative tail and the peak pressure was of the order of $3 \mathrm{MPa}$ peak to peak. Acoustic emissions were monitored by using an immersed unfocused ultrasound transducer with $10 \mathrm{MHz}$ central frequency and $3.175 \mathrm{~mm}$ diameter (V129RM, Olympus, Waltham, MA). The monitoring transducer was placed at an angle of $45^{\circ}$ from the axis of the shock wave [see Fig. 1(a)] at a distance of $\sim 1.5 \mathrm{~cm}$ from the focal region. The emissions were digitally filtered using a 7-20 $\mathrm{MHz}$ bandpass filter (third-order Butterworth), to attenuate the signal from the shock source and its reflections. LABVIEW 2015 (National Instruments, Austin, TX, USA) was used for all the data acquisition and analysis requirements.

A custom-built upright epifluorescent microscope, with an objective with $1.3 \mathrm{~cm}$ working distance (LMPLFLN $20 \times$ Objective, Olympus, Tokyo, Japan), was placed above the free surface of the test chamber and the focus of the objective was aligned with the focus of the shock waves [Fig. 1(a)]. The optical field of view (FOV) was of the order of $400 \mu \mathrm{m} \times 400 \mu \mathrm{m}$. For fluorescence detection, the FOV was illuminated by an ultraviolet light-emitting diode (UV LED) (central wavelength $385 \mathrm{~nm}$ and bandwidth $10 \mathrm{~nm}$, catalog
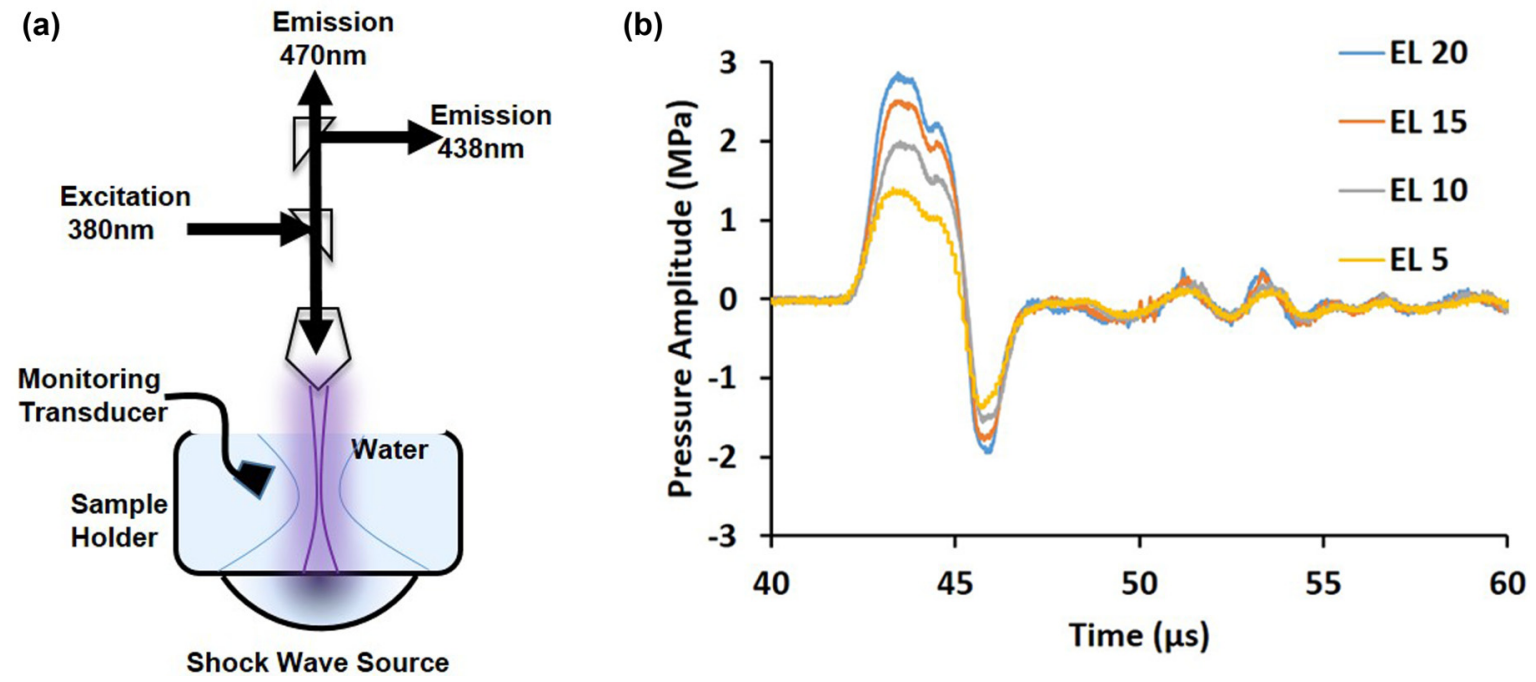

FIG. 1. Experimental setup. (a) The shock wave source is coupled to a temperature controlled water tank. The optical system consists of a LED light source $(380 \mathrm{~nm})$ which is focused by an objective in the water, coincident with the shock source, and fluorescent signals are detected by two photomultiplier tubes recording simultaneously at two different wavelengths (438 and $470 \mathrm{~nm}$ ). An unfocused monitoring transducer is used to detect acoustic emissions from the vicinity of the focal region. During experiments multilamellar lipid vesicles (MLVs), embedded with the dye Laurdan, are present everywhere in the tank. (b) Measured focal pressure waveforms for shock waves at four different energy settings. 

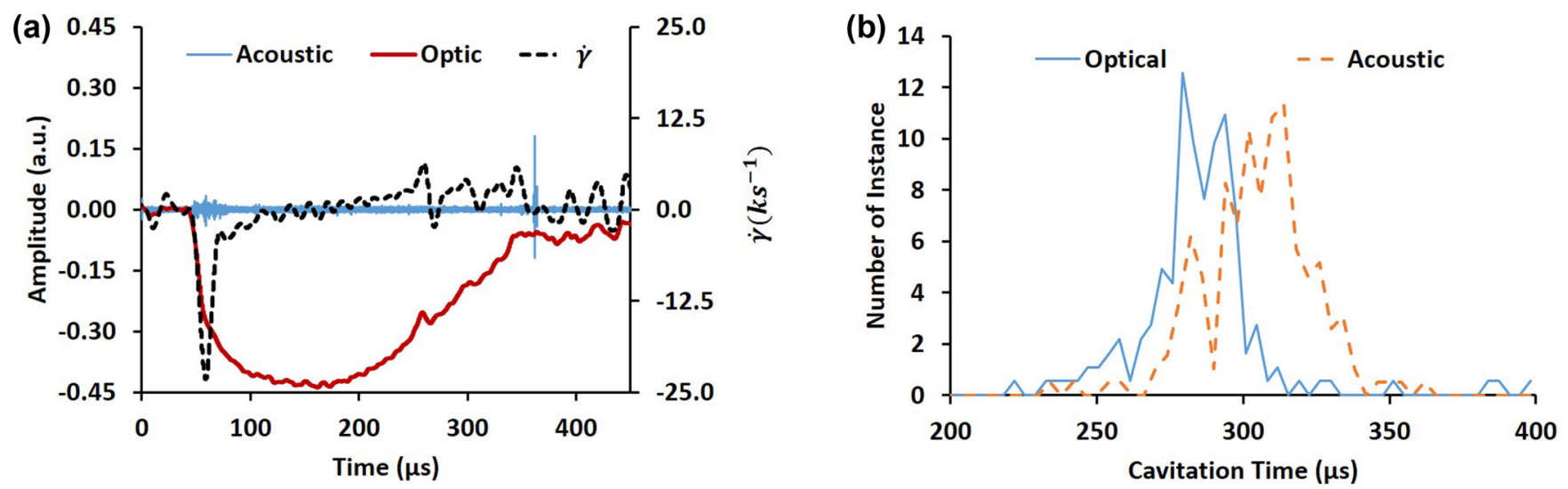

FIG. 2. (a) Optical intensity $\frac{\Delta I}{\mathrm{I}}$ at $470 \mathrm{~nm}$, acoustic emissions, and rate of deformation $\dot{\gamma}$ measured during a single cavitation event in DOPC suspension at $20^{\circ} \mathrm{C}$ in response to a shock impulse of energy level 20 . The shock wave arrives at $50 \mu$ s resulting in an acoustic emission, a drop in optical intensity, and a peak of rate of deformation. The cavitation bubble/cluster grows to a maximum extent at $170 \mu$ s and then collapses at around $350 \mu$ s producing a strong acoustic emission and a knee in the optical intensity. (b) The distribution of cavitation time for 200 such shock waves as determined from the acoustic and optical signals.

No. M385LP1, Thorlabs, Newton, NJ, USA) and the emitted fluorescence intensity was measured simultaneously by two photomultiplier tubes (HT493-003, Hamamatsu, Japan), $I_{438 \mathrm{~nm}}$ (filter at $438 \pm 12 \mathrm{~nm}$ ) and $I_{470 \mathrm{~nm}}$ (filter at $470 \pm$ $11 \mathrm{~nm})$, with a signal bandwidth of $8 \mathrm{MHz}$.

The measured optical signal was evaluated as $\frac{\Delta I}{I_{0}}=\frac{I(t)-I_{0}}{I_{0}}$, where $I_{0}$ is the initial emission intensity and it depends on the thermodynamic state of the interface, the concentration of dye molecules, and the design characteristics of the optical setup, e.g., focal volume. The rate of mechanical deformation induced by the shock wave was characterized by $\dot{\gamma}=\frac{d}{d t} \frac{\Delta I_{470 \mathrm{~nm}}}{I_{470 \mathrm{~nm}}}$. The thermodynamic state of the membrane was characterized by $\frac{\Delta R P}{R P_{0}}=\frac{\Delta I_{438 \mathrm{~nm}}}{I_{438 \mathrm{~nm}}}-\frac{\Delta I_{470 \mathrm{~nm}}}{I_{470 \mathrm{~nm}}}$ which, due to normalization, is insensitive to the amplitude of the light intensity [7]. For small perturbations $\frac{\Delta R P}{R P_{0}} \propto \Delta h_{i}$, i.e., change in the enthalpy of the interface [7].

\section{RESULTS}

Figure 2 shows the acoustic emission, optical signal $\left(\frac{\Delta I}{I_{0}}\right)$, and the mechanical deformation rate $(\dot{\gamma})$ measured from a DOPC suspension in response to a single shock wave. The shock wave was triggered at $t=0 \mu \mathrm{s}$ and arrived at the focus at around $t_{0}=50 \mu \mathrm{s}$, resulting in (i) a rapid drop in the optical signal, indicating formation of bubbles; (ii) an acoustic emission, which was detected at $60 \mu$ s due to the $10-\mu$ s propagation time to the monitoring transducer; and (iii) a negative spike in the rate of deformation. The optical signal continued to drop until about $170 \mu$ s after which it started to increase until it "recovered" at $t_{r}=350 \mu \mathrm{s}$, although not to baseline. The optical signal $\frac{\Delta I}{I_{0}}$ is consistent with Mie scattering based optical measurements during similar cavitation experiments (see, e.g., Fig. 7 in Ref. [20]), including the incomplete recovery of the optical signal at $t_{r}=350 \mu \mathrm{s}$, indicating that $\frac{\Delta I}{I_{0}}$ is related to the size of the bubble cluster. The time of recovery corresponds to a second, stronger acoustic emission at $t=360 \mu \mathrm{s}$, which is consistent with the collapse of a bubble or bubbles [20]. This double acoustic signature is characteristic of the creation and collapse of a bubble or bubble cluster in response to a shock wave and the time between the signals $t_{c}$ is an indicator of the intensity of the cavitation $[21,22]$. The lack of a distinct peak in $\dot{\gamma}$ at recovery, similar to the one observed at $t_{0}=50 \mu \mathrm{s}$, suggests that the observed decay in fluorescence signal is most likely dominated by lipid relaxation and diffusion, which is different from the collapsing cavity.

The cavitation time $t_{c}=t_{r}-t_{0}$ was determined from the optical signals where $t_{0}=50 \mu \mathrm{s}$ is the arrival time of the shock wave and $t_{r}$ was defined as the time when $\frac{\Delta I}{I_{0}}$ returned above a value of -0.1 , in the time window $t>90 \mu \mathrm{s}$, to ensure the initial emission was complete. If $\frac{\Delta I}{I_{0}}<-0.1$ at $t=90 \mu \mathrm{s}$ then it was assumed that there was no detectable cavitation. As shown in Fig. 2(b), both optical and acoustic detection provide a consistent estimate of cavitation time and in what follows only optically observed cavitation times have been reported.

In Fig. 3, the cavitation time is reported as a function of shock wave energy for MLVs in four different initial states, as indicated by the corresponding reduced temperature $T^{*}$. In all cases, cavitation time increases monotonically with increasing energy level. This is consistent with shock wave induced cavitation measured in water [21]. For the two formulations in a condensed state $\left(T^{*}<0\right), t_{c}<200 \mu$ s on average, whereas for the two formulations in a fluid state $\left(T^{*}>0\right), t_{c}>250 \mu \mathrm{s}$. This suggests that the duration of the cavitation is dependent on the thermodynamic state of the lipid membrane, with the longer cavitation times observed for more fluid initial states. Note that from the number of cavitation events plotted in Fig. 3, the presence of significant cavitation at Energy Level (EL) 5 and 8 for lipids close to gel-fluid phase transitions $\left(T^{*}=-0.045\right.$ and $\left.T^{*}=0.046\right)$ indicates the cavitation threshold is lower near the transition (see Fig. S1 in the Supplemental Material (SM) [23]). Therefore, this effect was investigated in more detail.

The effect of the thermodynamic state of lipid nanoparticles on cavitation threshold was determined by determining the cavitation rate (fraction of shock waves that result in detectable cavitation) as a function of energy level. Figure 4 shows the effect of different lipids at the same temperature and 

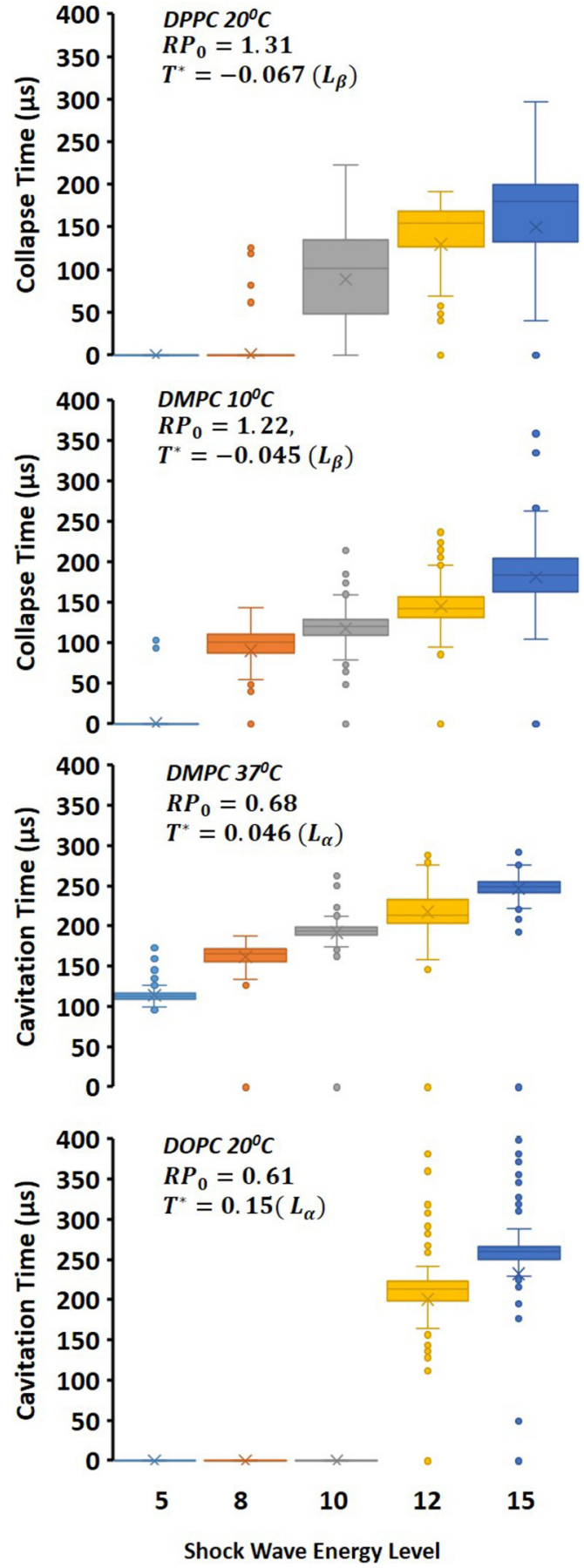

FIG. 3. Dependence of cavitation time as a function of shock wave energy level for four different initial lipid states. The initial state ranges from the condensed state, $T^{*}=-0.067$ (top) to the fluid state, $T^{*}=0.15$ (bottom). A cavitation time of zero indicates the absence of cavitation. For the gel initial state $T^{*}=0.15$, cavitation starts at EL 10 and the cavitation time increases with EL to a maximum of $200 \mu \mathrm{s}$. At $T^{*}=-0.045$ significant cavitation occurs for EL 8 and cavitation time increases with EL but again does not exceed $200 \mu \mathrm{s}$. At $T^{*}=0.046$, the threshold is lowered and cavitation occurs at all energy levels settings with a maximum cavitation time of 250 us. For $T^{*}=0.15$ cavitation starts at EL 12 and the cavitation time exceeds $250 \mu$ s at the highest setting. Dots indicate the outliers that are more than 1.5 times the upper/lower quartile.

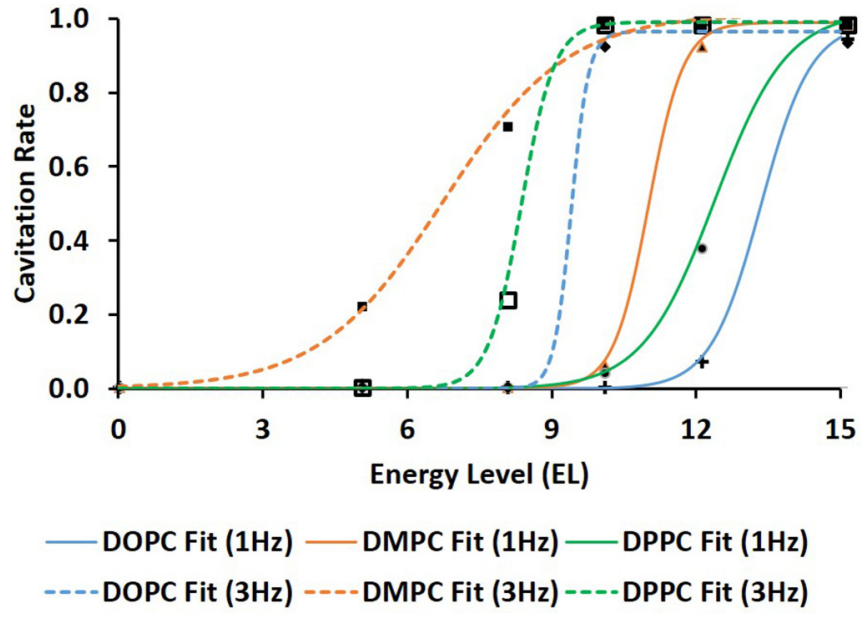

FIG. 4. Cavitation rate of aqueous suspension of liposomes measured as a function of the energy level, the thermodynamic state, and the repetition rate. Cavitation rate defined as number of cavitation events per 100 shocks has been plotted at two different shock repetition rates $1 \mathrm{~Hz}$ (solid curves) and $3 \mathrm{~Hz}$ (dashed curves). The markers show data and the curves show fitted sigmoids. Three different liposome suspensions were tested. The mean hydrodynamic radius and polydispersity index were as follows: DOPC $(160 \mathrm{~nm}$, 0.2), DMPC (140 nm, 0.17), and DPPC (452 nm, 0.44). All samples were tested at a temperature of $24^{\circ} \mathrm{C}$ and $p \mathrm{H} 7$.

pressure on the cavitation threshold. The cavitation rate was measured based on 100 shocks at five different energy levels and dose response fits were obtained. For each preparation the energy level was ramped from 5 to 15 at two different pulse repetition frequency (PRF)s of 1 and $3 \mathrm{~Hz}$. It can be seen that at both PRFs, the lipid preparation with $T^{*}$ closest to zero, i.e., DMPC, has the lowest threshold, further supporting the concept that the nucleation threshold is indeed lowest when the lipid is close to a phase transition.

Figure 5 shows the evolution of $\frac{\Delta R P}{R P_{0}}$ and $\frac{\Delta I}{I_{0}}$ for the four different initial states. Due to the low signal-to-noise ratio the traces are averaged over 500 shocks. Note the shock waves' energy levels are not the same but correspond to the energy level at which "consistent cavitation" was observed, defined here as obtaining a single peak in the $t_{c}$ histogram and $|\dot{\gamma}|>$ $3 \times 10^{-4} \mathrm{~s}^{-1}$. It can be seen that for all initial states, $\frac{\Delta R P}{R P_{0}}$ and $\frac{\Delta I}{I_{0}}$ have similar dynamics; that is, they are responding to the growth and collapse of cavitation.

However, for an initial gel state $\left(T^{*}<0\right)$ the peak is lower with $\frac{\Delta R P}{R P_{0}}<0.15$ whereas for the initial fluid state $\left(T^{*}>0\right)$, the peak $\frac{\Delta R P}{R P_{0}}$ is much larger $\left(\frac{\Delta R P}{R P_{0}} \approx 0.3\right)$. Based on the interpretation of the Laurdan spectrum, which will be discussed below, a $\frac{\Delta R P}{R P_{0}}>0$ corresponds to condensation of the lipid interface. Therefore, these observations suggest that during the expansion of the cavitation cluster, the lipids undergo condensation. Furthermore, when the condensation is more pronounced the lipids are initially in a fluid state. The significance of the quantitative differences in the response as a function of $T^{*}$ will be discussed in more detail below. Note that $\left|\frac{\Delta I}{I_{0}}\right|$ does not capture these state differences, as the signal is dominated by the appearance of gaseous scatterers in the FOV. In fact to a first order, the trend with $\left|\frac{\Delta I}{I_{0}}\right|$ would indicate 

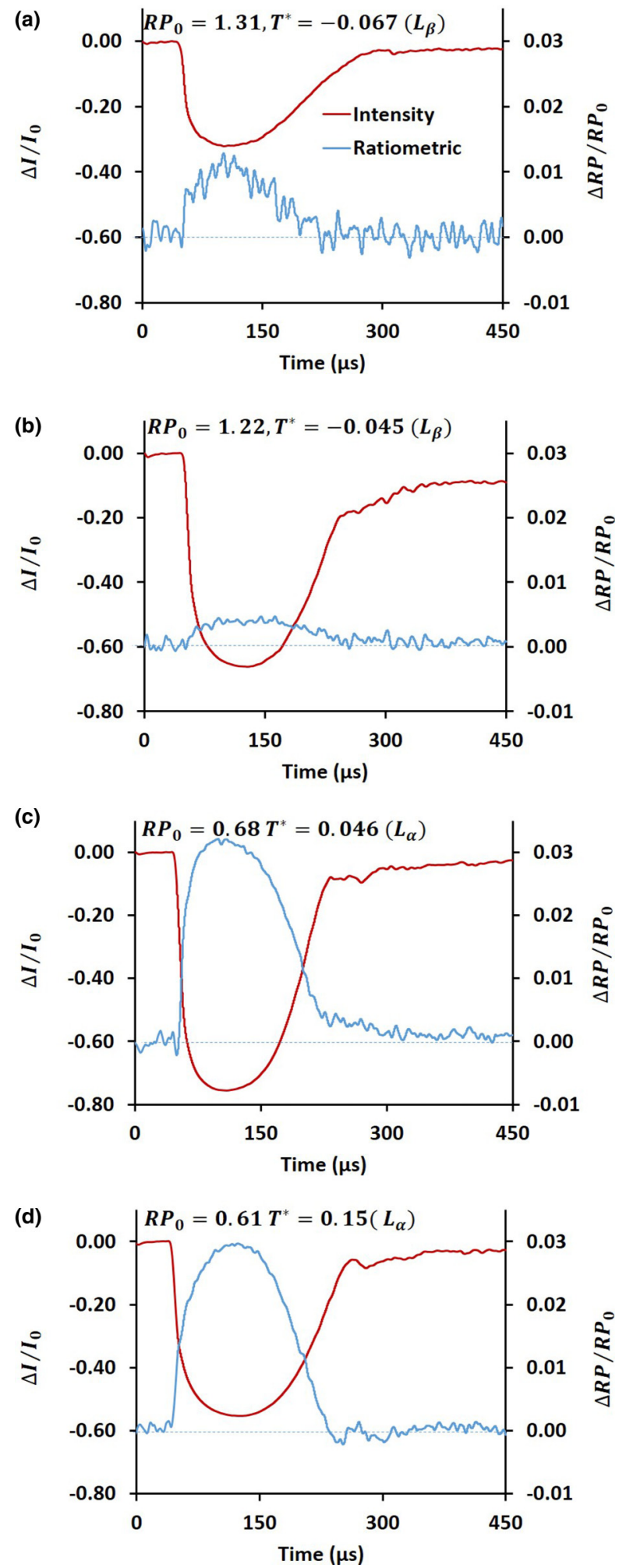

FIG. 5. Optical intensity $\frac{\Delta I}{I}$ and ratiometric parameter $\frac{\Delta R P}{R P_{0}}$ are plotted as a function of time for four initial conditions. Lipids and temperature give the initial condition, which is also reported by $R P_{0}$, These are (a) DPPC, $T=20^{\circ} \mathrm{C}$, EL 15; (b) DMPC, $T=10^{\circ} \mathrm{C}$, EL 15; (c) DMPC, $T=37^{\circ} \mathrm{C}$, EL 10 ; (d) DOPC, $T=20^{\circ} \mathrm{C}$, EL 12. The optical intensity change is greatest when $T^{*}$ is closest to zero indicating that the cavitation bubbles/cluster attains the greatest volume when the lipid is close to a transition. $\frac{\Delta R P}{R P_{0}}$ is greater for lipids in the fluid state than in the gel state, indicating a greater state change. that the fraction of such scatter is minimum for $T^{*}=-0.067$, $\left|\frac{\Delta I}{I_{0}}\right| \approx 0.3$; it peaks for $T^{*}=0.046,\left|\frac{\Delta I}{I_{0}}\right| \approx 0.75$, and then decreases again for $T^{*}=0.15,\left|\frac{\Delta I}{I_{0}}\right| \approx 0.55$. This is consistent with the observed lowering of cavitation threshold near a phase transition, which would imply more nucleation sites for the given field of view.

As the shock wave energy was increased there was an increase in $\dot{\gamma}$ as shown in Figs. 6(a) and 6(b). This is consistent with a more violent expansion of the cavity. However, the peak $\left|\frac{\Delta R P}{R P_{0}}\right|$ remained at $\sim 0.15$, suggesting a saturation of the change in the thermodynamic state of the interface during rapid expansion, a phenomenon which is common in such nonlinear systems [24].

A bimodal distribution in the cavitation time $t_{c}$ was often observed for EL 12 and 15; an example is shown in Fig. S2 in the SM [23], which suggests that the cavitation occurred in two distinct paths in the state diagram. To examine the difference in the two paths, the set of EL 12 waveforms show in Fig. S2 in the SM [23] was divided into two populations by using a cutoff of $t_{r}=270 \mu \mathrm{s}$. Figures 7(a) and 7(b) shown averaged traces for $\frac{\Delta R P}{R P_{0}}$ and $\dot{\gamma}$ for the two populations. It can be seen for $t_{r}>270 \mu$ s that $\frac{\Delta R P}{R P_{0}}$ is similar to Fig. 5 and the increase in $\frac{\Delta R P}{R P_{0}}$ suggests the lipid is being condensed. However, quite remarkably for $t_{r}<270 \mu \mathrm{s} \frac{\Delta R P}{R P_{0}}$ has the opposite sign suggesting that the lipid is becoming more fluid; it can be seen that this correlates with a relatively low $\dot{\gamma}$. These results suggest that when the cavity has a high expansion rate it condenses the lipid interface $\left(\frac{\Delta R P}{R P_{0}}>0\right)$, but when it has a low expansion rate the lipid interface is fluidized $\left(\frac{\Delta R P}{R P_{0}}<0\right)$. This insight is only available through the use of Laurdan and by quantifying the changes in its emission spectrum in terms of $\frac{\Delta R P}{R P_{0}}$. The effects can also be seen in lipids that were in gel phase Fig. S3 in the SM [23]. Finally, it was observed that $R P_{0}$ returned to the same initial state before a new shock wave was fired and there were no significant cumulative effects of a sequence of shock waves fired at a given PRF (Fig. S4 in the SM [23]).

\section{DISCUSSION}

The experimental results show that for an aqueous-lipid suspension, the characteristic cavitation features such as threshold and cavitation lifetime, as well as the thermodynamic state of the interface during cavitation, are dependent on the initial state of the lipid interface with the reduced temperature $T^{*}$ being a strong indicator of nucleation threshold and subsequent evolution of the cavity. Here we interpret these results in terms of thermodynamic principles to arrive at a phenomenological description of the observation and possible microscopic states of the lipid-water interface during cavitation. Our data showed that the cavitation threshold was lowest at phase transition $\left(T^{*} \approx 0\right)$; this is not consistent with the usual explanation of cavitation threshold in the presence of lipids or fats, which is attributed to the lowering of surface tension alone. At a given temperature different lipids should have a similar surface tension [25-27]. Furthermore, the surface tensions should decrease monotonically with temperature [28], and so a monotonic decrease in threshold would have been expected. Instead we observe a minimum at the phase 
transition temperature. We propose that the reduced cavitation threshold is because of the maximum in thermodynamic fluctuations that occur at the phase transitions. Under the assumption of local equilibrium, the amplitude of microscopic fluctuations is related to the second derivative of the thermodynamic potential of the system (see the Appendix). The enthalpy fluctuations of the lipids $\left|\left(\delta H_{2}\right)^{2}\right|$ are given by

$$
\left|\left(\delta H_{2}\right)^{2}\right|=k C_{p_{2}} T^{2},
$$

where $k$ is the Boltzmann constant, $T$ is the temperature, and $C_{p_{2}}$ is the heat capacity of the lipids at constant pressure. The heat capacity peaks at phase transition and so the fluctuations in enthalpy also have a maximum. The fluctuations in the volume of water $V_{1}$ (liquid and vapor) are related to the enthalpy of the lipids $\mathrm{H}_{2}$ (see the Appendix) as

$$
\left|\delta V_{1} \delta H_{2}\right| \approx \frac{k T^{2}}{P / C_{p_{2}}-T \frac{\partial P}{\partial H_{2}}},
$$

where $P$ is the equilibrium pressure. As described in the Appendix, the right-hand side of Eq. (2) also has a maximum at the transition. Therefore a peak in $\left|\left(\delta \mathrm{H}_{2}\right)^{2}\right|$ at phase transition due to a peak in $C_{p_{2}}$ also implies a peak in $\left|\left(\delta V_{1}\right)^{2}\right|$ of interfacial water at the phase transition of the lipid.

Our data are consistent with the hypothesis that the peak in these fluctuations gives rise to the minimum in cavitation threshold. In water, vaporization occurs when pressure drops below the saturation limit or the water-vapor boundary in the phase diagram. The vaporization process involves a finite relaxation time and if the pressure drop occurs faster than this relaxation time, such as during a shock wave, the system goes into the metastable regime of the water-vapor phase diagram. In the metastable regime while the system is in mechanical equilibrium with respect to the tensile stress, it is not in chemical equilibrium with respect to the water-to-vapor transition (partial equilibrium).

Equation (2) can be extended to the metastable regime under the assumption of local equilibrium (see the Appendix) where the heat capacity and compressibility of water are defined as functions of the dynamic local state. Therefore, as the system moves further into the metastable regime (increasing heat capacity, compressibility of water), thermal fluctuations also increase, eventually causing nucleation, which also results in a local minimum in the $P-V$ curve (spinodal boundary). Beyond this minimum, $\frac{\partial P}{\partial V_{1}}>0$ (compressibility of water is negative), which results in thermodynamic instability and sudden macroscopic expansion and phase change that takes place even in the absence of an external stress. Equation (2) is not valid beyond this point as the assumption of reversibility is not valid. The water-to-vapor phase change is accompanied by absorption of latent heat and dissipation due to nonequilibrium heat transfer, which establishes the chemical equilibrium [29]. The experimentally observed expansion phase (Fig. 2) (starting at $t=50 \mu \mathrm{s}$ ) is assumed to represent this process. Therefore, during the observed cavitation expansion the emission spectrum of the dye only measures $\delta H_{2}$, but $\delta V_{1}$ cannot be estimated using Eq. (2) due to the irreversible nature of the process.

In the data shown here (Table I), a rapid expansion of the vapor cavities resulted in condensation of lipids. Previously it has been shown that the coupling between the bulk and interface decreases with decreasing timescales of interactions $[33,34]$. In our case this implies that if the surrounding water cannot exchange heat with the interface then the heat of vaporization has to come from within the interface. This will result in a decrease in enthalpy of the lipids at the interface and hence their condensation $[35,36]$. This interpretation is further supported by the observation that at slower expansion rates, when there is sufficient time for the surrounding water to provide heat to the interface, the lipids get fluidized upon increase in the volume (surface area) of the cavity. The state of the lipids will also place constraints on the expansion of the cavity. For example, the cavitation time (Fig. 3) was shortest for the gel state, which presumably limits the amount of heat that can be transferred from the lipids to water for vaporization.

Evidence for the phase transition in lipids is being captured by $\triangle R P / R P_{0(1 \rightarrow 2)}$ which the experiments show is proportional to corresponding enthalpies of transitions [7,32,37]; i.e.,

$$
\Delta R P / R P_{0(1 \rightarrow 2)} \propto \Delta h_{(1 \rightarrow 2)}
$$

Table II shows the ratio of the peak of the $\triangle R P / R P_{0}$ and the ratio of the enthalpy of transition (from Table I). It can be seen that the ratios agree to within about $15 \%$ which is consistent with the hypothesis that lipids are indeed condensing to the $L_{c}$ phase during the expansion phase of cavitation. allowing for the fact that the literature values for enthalpy change are for quasistatic processes. This confirms that $\triangle R P / R P_{0}$ measures the state changes even during cavitation and not just during

TABLE I. Thermodynamic data associated with state changes in different lipid systems as reported in the literature [30] and corresponding spectral shift in Laurdan $\left|\frac{\Delta R P}{R P_{0}}\right|$ as observed in this study. $T_{m}$ is the chain melting temperature, $T$ is the temperature of the experiment, and $T^{*}$ is the corresponding reduced temperature, all at atmospheric pressure. Possible phase changes from the initial condition and the corresponding transition temperature $\left(T_{t}\right)$ as well as enthalpies $(-\Delta h)$ are listed. A range of values has been reported for phase transitions of DOPC suspension in water due to experimental difficulties in measuring subzero transitions in water [31]. $L_{\beta} \rightarrow L_{c}$ in DMPC, on the other hand, has not been studied as reliably as the other values reported here, because of the highly metastable nature of the transition $[30,32]$.

\begin{tabular}{cccccccccc}
\hline \hline Lipid & $T_{m}$ & $T$ & $T^{*}$ & Initial phase & $R P_{0}$ & $\left|\frac{\Delta R P}{R P_{0}}\right|$ & Phase change & $T_{t}$ & $-\Delta h\left(\frac{\mathrm{kJ}}{\mathrm{mol}}\right)$ \\
\hline DOPC & $-40^{\circ} \mathrm{C}$ & $20^{\circ} \mathrm{C}$ & $\sim 0.15$ & $L_{\alpha}$ & 0.622 & 0.029 & $L_{\alpha} \rightarrow L_{c}$ & $-12^{\circ} \mathrm{C}$ & $\sim 32-65$ \\
DMPC & $23.6^{\circ} \mathrm{C}$ & $37^{\circ} \mathrm{C}$ & 0.046 & $L_{\alpha}$ & 0.726 & 0.032 & $L_{\alpha} \rightarrow P_{\beta}$ & $23.6^{\circ} \mathrm{C}$ & 25.0 \\
& & $20^{\circ} \mathrm{C}$ & -0.01 & $P_{\beta}$ & 0.950 & 0.014 & $P_{\beta} \rightarrow L_{c}$ & $11.4^{\circ} \mathrm{C}$ & 15.8 \\
& & $10^{\circ} \mathrm{C}$ & -0.045 & $L_{\beta}$ & 1.208 & 0.004 & $L_{\beta} \rightarrow L_{c}$ & NA & NA \\
DPPC & $41.5^{\circ} \mathrm{C}$ & $20^{\circ} \mathrm{C}$ & -0.067 & $L_{\beta}$ & 1.300 & 0.012 & $L_{\beta} \rightarrow L_{c}$ & $18.3{ }^{\circ} \mathrm{C}$ & 14.2 \\
\hline \hline
\end{tabular}


TABLE II. The ratio of maximum amplitudes of the observed $\triangle R P / R P_{0}$ for different samples were calculated from the values in Table I. Assuming the final state as $L_{c}$, as discussed in the text, corresponding ratio of enthalpies calculated using Table I are also listed.

\begin{tabular}{lcccc}
\hline \hline & $(\mathrm{DMPC})_{\left(P_{\beta} \rightarrow L_{c}\right)}$ & $(\mathrm{DPPC})_{\left(L_{\beta} \rightarrow L_{c}\right)}$ & $(\mathrm{DOPC})_{\left(L_{\alpha} \rightarrow L_{c}\right)}$ & $(\mathrm{DMPC})_{\left(L_{\beta} \rightarrow L_{c}\right)}$ \\
\hline$\frac{\Delta R P / R P_{0(x)}}{\Delta R P / R P_{0(\mathrm{DMPC})}\left(L_{\alpha} \rightarrow L_{c}\right)}$ & 0.43 & 0.37 & 0.91 & 0.12 \\
$\frac{\Delta h_{(x)}}{\Delta h_{(\mathrm{DMPC})_{\left(L_{\alpha} \rightarrow L_{c}\right)}}}$ & 0.38 & 0.36 & $0.71-1.43$ & $\mathrm{NA}$ \\
\hline \hline
\end{tabular}

small acoustic perturbation as shown before [7]. We were unable to identify data in the literature for the (DMPC) ${\left.L_{\beta} \rightarrow L_{c}\right)}$ enthalpy change, but based on the measurements here we can predict it to be of the order of $-5 \frac{\mathrm{kJ}}{\mathrm{mol}}$.

Now to understand the microscopic state of the lipid interface during the process it is important to recall that phase transition in lipid interfaces involves several degrees of freedom with distinct timescales and enthalpy of conformational change; see [37] for a detailed overview. The timescales of the observed spectral shift in this study are of the order of $10-100 \mu \mathrm{s}$ which would correspond to formation of rotational isomers or gauches in the hydrocarbon tails of the lipid molecules and constitute the observed enthalpy change. In the literature a positive $\triangle R P / R P_{0}$, i.e., a blueshift in the Laurdan spectrum, is usually attributed to an increase in the packing of lipids. Two observations from our data are not consistent with this interpretation. First, we generally saw a blueshift during rapid bubble expansion when one would expect the packing to decrease as the surface area of the bubble increases. Second, we observe a saturation effect (recall Fig. 6) where cavities driven with stronger shock waves exhibited no increase in $\triangle R P / R P_{0}$ despite the fact the cavities appeared to grow to a larger size. Instead, the saturation is consistent with the spectral shifts being associated with the thermodynamic state of the lipid. The $\triangle R P / R P_{0}$ is dominated by the enthalpy associated with the phase transition (Table II); therefore, once lipids have condensed to a gel phase, any further expansion of the bubble would not lead to substantial change in $\triangle R P / R P_{0}$.

We have investigated the role of the thermodynamic state of the interface during cavitation. There are two aspects to the role of the thermodynamic state of the interface during acoustic cavitation: (i) the microscopic or statistical physics of nucleation of a vapor cavity at an interface and its effect on cavitation threshold; and (ii) the macroscopic state of the interface during rapid expansion of the cavity, in particular condensation.

The thermodynamic approach explains the role of the state of the interface in nucleation statistics without making any assumption about the microscopic structure of the cavity. An alternative bottom-up approach, for example, assuming a physical cavity between the bilayer leaflets [38], has a major challenge in properly accounting for the energy required for the removal of interfacial water, which is closely tied to compression and condensation of the lipids to the $L_{c}$ phase [32,39-41]. Our approach only asserts that nucleation is most likely at the site of maximum fluctuations; however, it is not clear that the hydrophobic core would be that site and not the water-lipid interfacial region, for example [42].

Similarly, during macroscopic expansion of the cavity, bottom-up models have intuitively assumed increase in surface tension upon expansion; however, as discussed, cavitation induced dehydration would result in compression or increase in lateral pressure and hence a decrease in surface tension [27]. Finally, apart from compression, our study also highlights the importance of the dehydration and jamming of lipids at the expanding interface. Such effects have also been shown to cause reduction in intralamellar distances and compression in MLV suspensions [36,43], which has also informed our vision of the phenomenon.

Our vision of the cavitation phenomenon at the interface deduced from the observations reported here is sketched in Fig. 8. The cavitation is preferentially nucleated at a lipid interface, due to increased fluctuations. Once formed, the cavity grows under the negative acoustic pressure and more lipid is incorporated into the interface. This lipid interface provides the enthalpy for vaporization of water molecules into the cavity resulting in the lipid at the interface condensing to the $L_{c}$ gel phase.
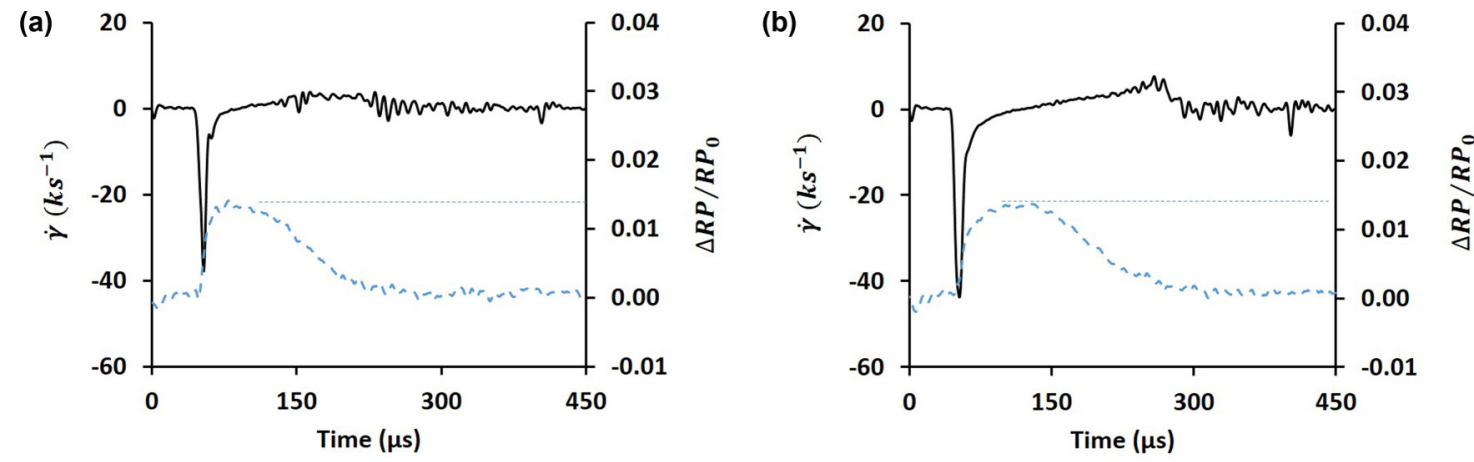

FIG. 6. $\triangle R P / R P_{0}$ and $\dot{\gamma}$ averaged over 500 shocks at energy levels (a) 12 and (b) 15 for DMPC suspension at $23^{\circ} \mathrm{C}$. At the higher energy level the growth rate $\dot{\gamma}$ (solid line) was larger, indicating the cavitation was driven harder, but the $\Delta R P / R P_{0}$ (dashed line) plateaued to the same value, suggesting the lipid obtained the same condensed state. 
(a)

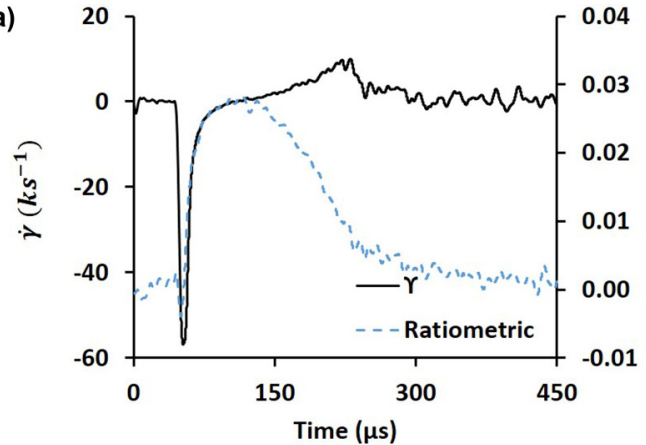

(b)

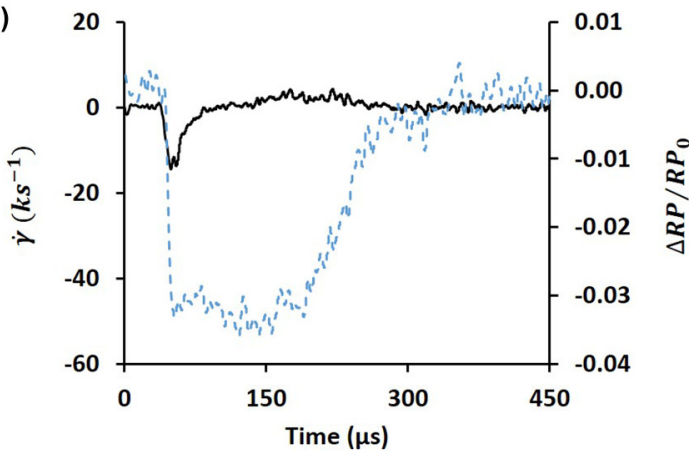

FIG. 7. The rate of mechanical deformation $(\dot{\gamma})$ and $\Delta R P / R P_{0}$ for a DMPC solution at $37{ }^{\circ} \mathrm{C}$ (a) waveforms averaged with $t_{r}>270 \mu \mathrm{s}$ gives high peak $\dot{\gamma}$, and $\frac{\Delta R P}{R P_{0}}>0$ indicates the lipid condenses during cavitation; (b) waveforms averaged with $t_{r}<270 \mu$ s give low peak $\dot{\gamma}$ while $\frac{\Delta R P}{R P_{0}}<0$ indicates the lipid fluidizing during cavitation. The distribution of $t_{r}$ is given in Fig. S2 in the SM [23].

We end by suggesting that there is no reason to assume that lipids are necessary for a condensation event to happen during the rapid expansion of a cavity. Similar thermodynamic considerations should also be relevant for the water-vapor interface during cavitation in the absence of lipids. In this regard, the present study may provide insight into the phenomenon of nucleation of ice during cavitation, also known as sonocrystallization, which has been proposed to explain experimentally observed nucleation of ice at the interface [44-46]. Based on seminal work by Hickling [47] it is generally accepted that sonocrystallization is due to adiabatic compression by shock waves emitted from a bubble collapse

(a)

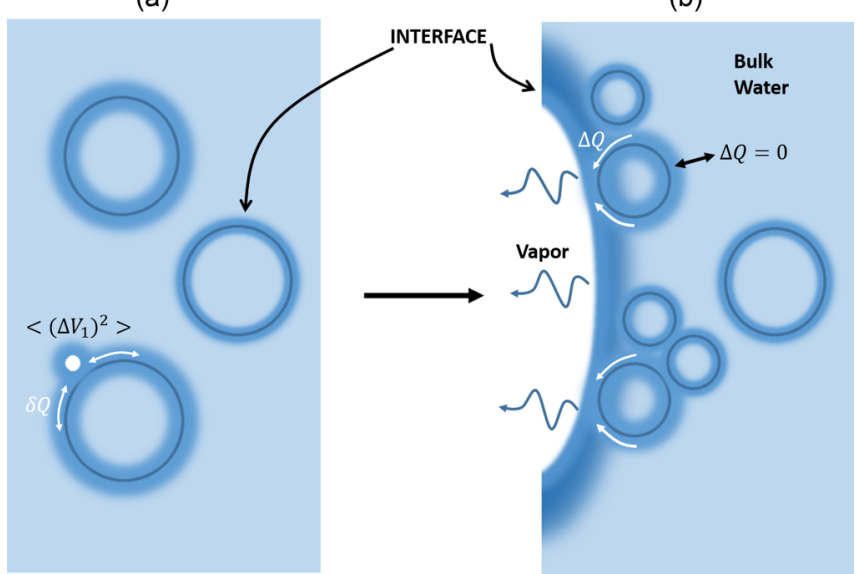

FIG. 8. Illustration of the proposed evolution of the interface during cavitation in a lipid environment. The interface is represented by the dense blue strip, the lighter shade the surrounding water, and white is the vapor cavity. (a) Due to reversible heat exchanges between lipids and interface water $(\delta Q)$, microscopic vapor cavities are formed at the interface of a liposome. (b) The initial cavity grows recruiting more lipid vesicles at the cavity interface. Water will be lost from the lipid as vapor to the cavity, which requires heat of vaporization, which is compensated by the irreversible heat flow ( $\Delta Q$ ) within the interface from lipids to water vapor (white arrows), whereas no heat flow from bulk to the interface (black arrow) due to dynamic decoupling of the interface (see text), resulting in condensation of the interface. and not from bubble expansion. At the time of Hickling's work there were only limited data on shock compression of water [48], and subsequent work has questioned the validity of a phase transition during shock compression [49] of up to several gigapascals. We hypothesize that if the interface of a cavity in water is adiabatically uncoupled from the bulk then the interface will cool down as it needs to provide the enthalpy for vapor to form. A sufficiently large decrease in the enthalpy could result in the water forming ice at the interface.

The production of ice can be estimated by breaking down the adiabatic expansion into an isentropic and an isothermal (dissipation) process as follows. If we assume that the vapor pressure inside the cavity drops isentropically to $100 \mathrm{~Pa}$ upon cavitation, then the temperature drops to approximately $T=$ $-20^{\circ} \mathrm{C}$, as seen on a temperature-entropy (TS) diagram of water-vapor-ice [50]. The entropy of vaporization of water is of the order of $6 \mathrm{~kJ} / \mathrm{kg} \mathrm{K}$, so assuming a $15 \%$ rate of entropy production (represented by an isothermal process) the entropy change will be $s-s_{0} \approx 0.9 \mathrm{~kJ} / \mathrm{kg} \mathrm{K}$, where $s_{0}$ is the entropy of water at $0{ }^{\circ} \mathrm{C}$ and $100 \mathrm{kPa}$. As can be seen on a water-vaporice TS state diagram [50], this amounts to approximately 4:1 ice-to-vapor mixture. Thus for every five water molecules entering the expanding boundary, four molecules will be deposited as ice at the interface [51] and one molecule will contribute to the volume of the expanding cavity. Similar to relaxation of condensed lipids observed in this study, the ice should also disappear in millisecond timescales [52], as the pressure equilibrates to initial conditions and water returns to the equilibrium liquid state. However, if the initial state is that of supercooled water (metastable liquid), upon nucleation of microscopic ice during the negative pressure tail, the crystal will expand into the surrounding supercooled water as the system will relax to the new equilibrium state of a macroscopic mixture of ice and water, as has been observed during sonocrystallization experiments [53].

In conclusion, the results here suggest that the thermodynamics of interfaces plays a crucial role in the nucleation and subsequent evolution of cavitation. The cavitation threshold was found to be related to the magnitude of microscopic fluctuations in the interface, which is a function of the thermodynamic susceptibility of the interface-governed by the presence of lipids in this study. The threshold for cavitation was found to be minimum when the interface is close to phase 
transition as the fluctuations are most intense at that point. Once nucleated, if the cavity underwent a fast expansion, the interface was adiabatically uncoupled from the bulk and the lipids cooled and condensed to a gel phase as water formed vapor inside the cavity. In the case of a slower expansion the interface received sufficient heat from the bulk to become more fluid and remain hydrated. The critical rate which separates these two phenomena was not resolved due to the limitations of the current experimental setup. The study also has important implications for applications emphasizing ultrasound and membranes, for example, drug delivery, acoustic neurostimulation, and ultrasound contrast agents where the role of interfacial entropy has not been incorporated in most models.

\section{APPENDIX: ON FLUCTUATIONS COUPLING AT THE INTERFACE}

In the following, we derive a relation explaining the observed minimum in the cavitation threshold (nucleation of vapor cavity in water) near a phase transition of the suspended lipids. Note that the derivation assumes nucleation results from reversible fluctuations, which is valid until the subsequent expansion of the cavity is irreversible. In homogenous nucleation theory, cavitation is regarded as a water-to-vapor phase transition under tensile stress [54]. Observed in the pressure-volume state diagram, the vapor phase becomes more stable once the pressure drops below the saturation boundary. However, the transition requires a finite time in order for the thermal fluctuations to overcome the entropy required to form the new interface (activation entropy, $\Delta S_{\text {act }}$ ). Therefore, if the system is subjected to tensile stress quasistatically, i.e., the system has sufficient time to relax, the vapor phase appears at the saturation boundary. However, dynamic tensile stress, such as during a shock wave, can result in metastable states (region between the saturation line and the spinodal) [24]. This is further complicated in the presence of lipids. As discussed below, a top-down approach is better suited to address such complexity as we have access to several macroscopic variables, starting from the shock wave induced pressure changes.

There are two main steps to understanding the effect of lipids on cavitation threshold in the presence of shock waves, (i) how fluctuations in specific observables associated with water are affected by fluctuations in specific observables associated with the lipids at the interface (fluctuation coupling), and (ii) how these fluctuations change in the presence of shock waves.

\section{Fluctuations in stationary water}

Let us first consider stationary water without any lipids. From statistical thermodynamics the probability $w$ of a given state scales exponentially with the magnitude of entropy variation, i.e., $w=e^{\frac{\left(S-S_{0}\right)}{k}}$, where $S_{0}$ is the equilibrium entropy and $k$ is the Boltzmann constant. The fluctuation in entropy is $\left|(\delta S)^{2}\right| \sim k C_{p}$, where $C_{p}$ is isobaric heat capacity [55]. The meaning of fluctuation in entropy is not trivial; here it means variations in the entropy as an analytical function $S\left(x_{1}, x_{2}, \ldots\right)$ due to fluctuations in the variables $x_{i}$ 's, which, in principle, are experimentally observable, such as volume, con-
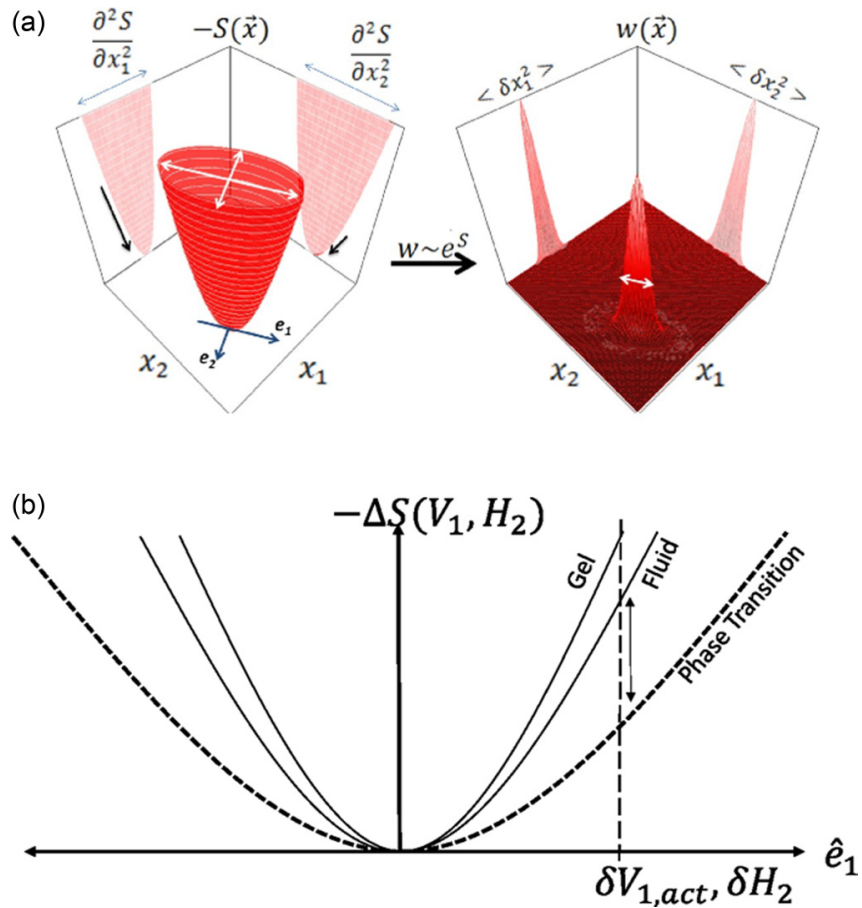

FIG. 9. (a) Generalized quadratic entropy potential for two experimental observables $S\left(x_{1}, x_{2}\right)$ [60]. The bases $x_{1}, x_{2}$ are not necessarily orthogonal; however, corresponding $e_{1}, e_{2}$ can be found that are orthogonal along the principle curvatures of the quadratic potential. The projections indicate how the susceptibility of a particular observable is related to principle curvatures of the potential. The probability distribution is obtained from the entropy potential $w \sim$ $e^{S}$, which gives fluctuations as the width of the distribution. Notice that for an arbitrary orientation of $e_{1}, e_{2}$ fluctuations as projected on $x_{1}, x_{2}$ will generally be coupled. (b) Projection of the potential on principal axis $\hat{e}_{1}$ shows the effect of change in state in terms of change in the curvature of the potential (thermodynamic susceptibility). Near phase transition, the decreased curvature implies higher probability such that $\sqrt{\left|(\delta V)^{2}\right|}>\delta V_{\text {act }}$ and hence lowered threshold.

centrations, energy of a part of the system, and charge [56]. Then $C_{p}$ measures the amount of heat that goes into changing $x_{i}$ 's reversibly for a unit change in temperature at constant pressure. Similarly, volume fluctuations are $\left|(\delta V)^{2}\right| \approx$ $k \beta_{T} V T$ where $\beta_{T}$ is isothermal compressibility. Cavitation becomes increasingly likely if the fluctuations are greater than the activation value; that is, $\sqrt{k C_{p}}>\delta S_{\text {act }}$ or equivalently $\sqrt{k \beta_{T} V T}>\delta V_{\text {act }}$. Figure 9 provides a visual representation of how these conditions affect nucleation statistics. While $\left|(\delta S)^{2}\right|$ represent variations on vertical axis, $\left|(\delta V)^{2}\right|$ result from the projection of the entropy potential $S\left(x_{1}, x_{2}, \ldots\right)$ on a horizontal axis, e.g., $x_{1}$, and $\beta_{T}$ is the projected curvature. Due to the Boltzmann principle, the system is exponentially more likely to be near the principle curvature, i.e., the system will on average fluctuate along the principle curvature. This is the reason why, near an equilibrium, it is not required to measure all other observables, i.e., $x_{2}, x_{3}, \ldots$, as $\left|(\delta S)^{2}\right|$ on average can be estimated based on any one of the $\left|\left(\delta x_{i}\right)^{2}\right|$ alone.

Note that $\delta V_{\text {act }}$ can be related to the critical radius $r_{c}$ in classical nucleation theory through $\delta V_{\text {act }}=\frac{4}{3} \pi r_{c}{ }^{3}$, where $r_{c}=$ $\frac{2 \sigma(T)}{\left(P_{l}-P_{v}\right)}\left(P_{v}\right.$ is the vapor pressure and $P_{l}$ is the pressure in liquid) 
is of the order of nanometers and is typically not accessible as an experimental observable. In comparison, $\sqrt{k \beta_{T} V T}$ and $\sqrt{k C_{p}}$ are macroscopic properties of the entire system that can, in principle, be measured during an experiment. This provides a top-down approach to nucleation statistics, with a foundation ensured by the second law of thermodynamics [57]. Note that in the presence of lipids, $\beta_{T}$ of the system undergoes a change and has a minimum near phase transition $[58,59]$, which is also the effective $\beta_{T}$ that should enter the fluctuation relation. Thus volume fluctuations in general will be maximized in the entire system, including those from (a) vapor cavities forming in water alone, (b) vapor cavities forming with lipids at the surface, and (c) pressure volume changes in lipids or water in the absence of cavities. Thus even on the basis of $\beta_{T}$ it is implicitly clear that the cavitation threshold will be lowered near phase transitions in the lipids. This can also be shown explicitly based on the coupling between the observables specific to lipids and water.

\section{Fluctuations in stationary lipid and water system}

When the system consists only of water, the macroscopic extensive variables have a simple meaning, which is directly related to the specific properties of water. In a mixture, however, this is not true as the macroscopic extensive variables will have contributions from different constituents. A typical approach is to represent them as a linear combination of the specific properties of the constituent, for example, $S\left(x_{i}\right)=$ $S_{1}\left(x_{i 1}\right)+S_{2}\left(x_{i 2}\right)=\alpha_{1} s_{1}+\alpha_{2} s_{2}$ where $\alpha_{i}$ 's can be the mole fractions. However, this intrinsically assumes that the fluctuations in the two systems are independent; i.e., $w\left(x_{1}, x_{2}\right)=$ $w\left(x_{1}\right) . w\left(x_{2}\right)$. In other words it ignores the contribution of the interface $S_{1,2}$. Therefore, it is more rigorous to not assume any rule for the combination, rather a general functional dependence $S\left(x_{1}, x_{2}\right)$. Examples of entropy potential defined as a function of the parts of the system are rare [56]; therefore, for the completeness and clarity of the derivation below, it should be noted, for example,

$$
V=V_{1}+V_{2} \Rightarrow(\partial V)_{V_{2}}=\left(\partial V_{1}\right)_{V_{2}} \Rightarrow \frac{\partial S}{\partial V_{1}}=\frac{\partial S}{\partial V}=-\frac{P}{T} .
$$

$P$ is equilibrium pressure in the entire system and $T$ is the temperature. The physical significance of the partial derivatives with respect to the parts of the system can be inferred from a thought experiment where a gas (subsystem 1) is trapped between a piston and an incompressible fluid (subsystem 2). Compressing the piston by $\partial V$ changes the entropy $\partial S$ of the system setting up a pressure through the entire system $\frac{\partial S}{\partial V}=-\frac{P}{T}$, note that $P$ then represents a change in pressure. However, the change in volume can primarily be traced to a change in the volume of the gas $\partial V_{1}$, which sets up the pressure $\frac{\partial S}{\partial V_{1}}$ in equilibrium with the rest of the fluid. Therefore, $\frac{\partial S}{\partial V_{1}}=\frac{\partial S}{\partial V}=-\frac{P}{T}$, as long as the system is in equilibrium.

${ }^{1}$ In our system, we have water, lipid membranes, and dye molecules. The dye molecules act as a reporter of the interface and so can be considered as a single "membrane" system [7]. Here we want to understand how the fluctuations in the observables specific to water (subsystem 1) are affected by the fluctuations in the observables specific to lipid membrane (subsystem 2). As seen in Fig. 9 and discussed above, the choice of $x_{1}, x_{2}$ (e.g., $\left.V_{1}, V_{2}\right)$ is arbitrary from a theoretical perspective. However, experimentally it should be based on what variables are accessible, given that choosing any $x_{i}$ does not affect the accounting of $S$. In our experimental setup $x_{1}$ is the volume of water (i.e., $V_{1}$ of subsystem 1) and $x_{2}$ is the enthalpy of lipids (i.e., $\mathrm{H}_{2}$ of subsystem 2), in which case expanding the entropy potential of the entire system near an equilibrium yields

$$
\begin{aligned}
S\left(V_{1}, H_{2}\right)= & S_{0}+\frac{1}{2} \frac{\partial^{2} S}{\partial V_{1}{ }^{2}}\left(\delta V_{1}\right)^{2} \\
& +\frac{\partial^{2} S}{\partial V_{1} \partial H_{2}} \delta V_{1} \delta H_{2}+\frac{1}{2} \frac{\partial^{2} S}{\partial H_{2}{ }^{2}}\left(\delta H_{2}\right)^{2} .
\end{aligned}
$$

Making use of $\frac{\partial S}{\partial V_{1}}=\frac{\partial S}{\partial V}$ as shown in (A1) the mixed derivative can be expressed as

$$
\frac{\partial^{2} S}{\partial V_{1} \partial H_{2}}=\frac{\partial}{\partial H_{2}} \frac{\partial S}{\partial V_{1}}=\frac{\partial}{\partial H_{2}}\left(-\frac{P}{T}\right)=\frac{\frac{P}{C_{p_{2}}}-T \frac{\partial P}{\partial H_{2}}}{T^{2}},
$$

where $C_{p_{2}}=\frac{\partial H_{2}}{\partial T}$ is the heat capacity of the lipid. Comparing the coefficient with a Gaussian distribution of two variables corresponding to $w=e^{\frac{\left(S-S_{0}\right)}{k}}$, where $S$ is given by Eq. (A2), we get

$$
\left|\delta V_{1} \delta H_{2}\right| \approx \frac{k T^{2}}{\frac{P}{C_{p_{2}}}-T \frac{\partial P}{\partial H_{2}}} .
$$

The relation shows how the enthalpy fluctuations $\left|\left(\delta H_{2}\right)^{2}\right|$ affect the volume fluctuations $\left|\left(\delta V_{1}\right)^{2}\right|$, which ultimately results in nucleation of vapor cavity. Furthermore, $\left|\left(\delta \mathrm{H}_{2}\right)^{2}\right| \approx$ $k C_{p_{2}} T^{2}$ which implies a maximum in $\left|\left(\delta H_{2}\right)^{2}\right|$ at the phase transition due to the corresponding maximum in $C_{p_{2}}$. Recognizing that $\frac{\partial P}{\partial H_{2}}$ was previously measured to be negative and minimum during phase transition (enthalpy of lipids increased during negative phase of small pressure perturbation [7]) implies that the right-hand side of Eq. (A4) is positive and maximum during phase transition. Hence $\left|\left(\delta V_{1}\right)^{2}\right|$ is maximum near a phase transition in lipids. The relation is valid as long as we can define heat capacity or compressibility locally as a function of dynamic pressure and temperature (i.e., the system is in local equilibrium), which is also going to allow their limited extension to shock wave induced state changes.

\section{Fluctuations during a shock wave}

While the above discussion assumed complete equilibrium, it can be extended to an arbitrary macroscopic state (partial equilibrium) [61] by observing the system for a time interval $\Delta t$ that is small compared to the relaxation time for the equilibrium. The entropy is then the sum of entropies of subsystems so small that the corresponding individual relaxation times are much smaller than $\Delta t$. Then during $\Delta t$ each subsystem can be assumed in their particular local equilibrium. For example, in our experiment a time resolution of $1 \mu \mathrm{s}$ and speed of sound $\sim 1500 \mathrm{~m} / \mathrm{s}$ gives the size of subsystem as $\sim 1.5 \mathrm{~mm}$, which is of the same order as the FOV of the optical setup. Furthermore, $1 \mu \mathrm{s}$ is significantly smaller than the cavitation timescale as well as the lateral diffusion timescales of the lipids. Indeed, we have previously shown that for subthreshold acoustic impulses of up to EL 
5 , the assumption of local equilibrium correctly predicts the response of lipids. That is, the acoustic response of lipids for subthreshold pressure pulses was maximum at phase transition where the heat capacity is maximum [7].

Thus, small acoustic perturbations can be imagined as the system oscillating in the entropy potential of Fig. 9 [62]. However, increasing the amplitude can then deform the potential itself (curvatures, i.e., heat capacity and compressibility, vary as a function of pressure). Indeed, as the shock wave expands the liquid into the metastable regime, compressibility increases and diverges as the liquid approaches vaporization spinodal [63,64], and since $\left|(\delta V)^{2}\right| \approx k \beta_{T} V T$, fluctuations diverge as well making nucleation unavoidable. The presence of lipids further contributes to these fluctuations as discussed, thus lowering the threshold for nucleation. Beyond nucleation the system becomes unstable resulting in irreversible macroscopic expansion of a cavity, due to nonequilibrium heat transfer, and the equations above are not valid anymore.

Data will be made available on Oxford Research Data Archive [65].

\section{ACKNOWLEDGMENTS}

We acknowledge the support by Engineering and Physical Sciences Research Council (EPSRC) under Programme Grant No. EP/L024012/1 (OxCD3: Oxford Centre for Drug Delivery Devices). We thank Prof. Lawrence Crum for a critical reading of the manuscript.
[1] D. C. F. Couzens and D. H. Trevena, Tensile failure of liquids under dynamic stressing, J. Phys. D: Appl. Phys. 7, 2277 (1974).

[2] P. P. Ye, J. R. Brown, and K. B. Pauly, Frequency dependence of ultrasound neurostimulation in the mouse brain, Ultrasound Med. Biol. 42, 1512 (2016).

[3] R. K. Schlicher, H. Radhakrishna, T. P. Tolentino, R. P. Apkarian, V. Zarnitsyn, and M. R. Prausnitz, Mechanism of intracellular delivery by acoustic cavitation, Ultrasound Med. Biol. 32, 915 (2006).

[4] J. Zhang, S. Shrivastava, and R. O. Cleveland, Rabbitts TH lipid-mRNA nanoparticle designed to enhance intracellular delivery mediated by shock waves, ACS Appl. Mater. Interfaces 11, 10481 (2019).

[5] J. Blackmore, S. Shrivastava, J. Sallet, C. R. Butler, and R. O Cleveland, Ultrasound neuromodulation; a review of results, mechanisms and safety, Ultrasound in Medicine and Biology (to be published).

[6] D. Li, A. Pellegrino, A. Hallack, N. Petrinic, A. Jérusalem, and R. O. Cleveland, Response of single cells to shock waves and numerically optimized waveforms for cancer therapy, Biophys. J. 114, 1433 (2018).

[7] S. Shrivastava and R. O. Cleveland, Schneider MF on measuring the acoustic state changes in lipid membranes using fluorescent probes, Soft Matter 14, 9702 (2018).

[8] I. Tasaki, E. Carbone, K Sisco, and I. Singer, Spectral analyses of extrinsic fluorescence of the nerve membrane labeled with aminonaphthalene derivatives, Biochim. Biophys. Acta 323, 220 (1973).

[9] E. Sackmann, Biological membranes: architecture and function, in Handbook of Biological Physics, Vol. 1, edited by R. Lipowsky and E. Sackmann (Elsevier, Amsterdam, 1995).

[10] E. A. Disalvo, F. Lairion, F. Martini, E. Tymczyszyn, M. Frías, H. Almaleck, and G. J. Gordillo, Structural and functional properties of hydration and confined water in membrane interfaces, Biochim. Biophys. Acta 1778, 2655 (2008).

[11] D. Marsh, Lateral pressure in membranes, Biochim. Biophys. Acta 1286, 183 (1996)

[12] A. Einstein, Conclusions drawn from the phenomena of capillarity, Ann. Phys. 4, 513 (1901).

[13] L. D. Landau and E. M. Lifshitz, Statistical Physics, 3rd ed., Part 1 (Butterworth-Heinemann, Burlington, MA, 1980), pp. $517-518$
[14] M. E. M. Azouzi, C. Ramboz, J.-F. Lenain, and F. Caupin, A coherent picture of water at extreme negative pressure, Nat. Phys. 9, 38 (2012).

[15] W. J Galloway, An experimental study of acoustically induced cavitation in liquids, J. Acoust. Soc. Am. 26, 849 (1954).

[16] B. M. Borkent, S. Gekle, A. Prosperetti, and D. Lohse, Nucleation threshold and deactivation mechanisms of nanoscopic cavitation nuclei, Phys. Fluids 21, 102003 (2009).

[17] A. Maxwell, C. Cain, T. Hall, B. Fowlkes, and Z. Xu, Probability of cavitation for single ultrasound pulses applied to tissues and tissue-mimicking materials, Ultrasound Med. Biol. 39, 449 (2013).

[18] A. A. Doinikov and A. Bouakaz, Review of shell models for contrast agent microbubbles, IEEE Trans. Ultrason. Ferroelectr., Freq. Control 58, 981 (2011).

[19] P. Marmottant, S. van der Meer, M. Emmer, M. Versluis, N. de Jong, S. Hilgenfeldt, and D. Lohse, A model for large amplitude oscillations of coated bubbles accounting for buckling and rupture, J. Acoust. Soc. Am. 118, 3499 (2005).

[20] T. J. Matula, P. R. Hilmo, B. D. Storey, and A. J. Szeri, Radial response of individual bubbles subjected to shock wave lithotripsy pulses in vitro, Phys. Fluids 14, 913 (2002).

[21] A. J. Coleman, M. J. Choi, J. E. Saunders, and T. G. Leighton, Acoustic emission and sonoluminescence due to cavitation at the beam focus of an electrohydraulic shock wave lithotripter, Ultrasound Med. Biol. 18, 267 (1992).

[22] C. C. Church, A theoretical study of cavitation generated by an extracorporeal shock wave lithotripter, J. Acoust. Soc. Am. 86, 215 (1989).

[23] See Supplemental Material at http://link.aps.org/supplemental/ 10.1103/PhysRevMaterials.3.055602 for different representations of the data presented in this manuscript, showing the minimum in cavitation threshold for lipids near phase transition, and the effect of expansion rate on different states and initial states of lipids.

[24] S. Shrivastava, K. H. Kang, and M. F. Schneider, Solitary shock waves and adiabatic phase transition in lipid interfaces and nerves, Phys. Rev. E 91, 012715 (2015).

[25] R. C. MacDonald and S. A. Simon, Lipid monolayer states and their relationships to bilayers, Proc. Natl. Acad. Sci. U. S. A. 84, 4089 (1987). 
[26] S. Feng, Interpretation of mechanochemical properties of lipid bilayer vesicles from the equation of state or pressure-area measurement of the monolayer at the air-water or oil-water interface, Langmuir 15, 998 (1999).

[27] F. Jähnig, What is the surface tension of a lipid bilayer membrane? Biophys. J. 71, 1348 (1996).

[28] O. Albrecht and H. Gruler, Polymorphism of phospholipid monolayers, J. Phys. 39, 301 (1978).

[29] M. Cowperthwaite and T. J. Ahrens, Thermodynamics of the adiabatic expansion of a mixture of two phases, Am. J. Phys. 35, 951 (1967).

[30] R. Koynova and M. Caffrey, Phases and phase transitions of the phosphatidylcholines, Biochim. Biophys. Acta, Rev. Biomembr. 1376, 91 (1998).

[31] S. Kaneshina, H. Ichimori, T. Hata, and H. Matsuki, Barotropic phase transitions of dioleoylphosphatidylcholine and stearoyl-oleoylphosphatidylcholine bilayer membranes, Biochim. Biophys. Acta, Biomembr. 1374, 1 (1998).

[32] H. H. Chang, R. K. Bhagat, R. Tran, and P. Dea, Subgel studies of dimyristoylphosphatidylcholine bilayers, J. Phys. Chem. B 110, 22192 (2006).

[33] T. Heimburg, Mechanical aspects of membrane thermodynamics. Estimation of the mechanical properties of lipid membranes close to the chain melting transition from calorimetry, Biochim. Biophys. Acta, Biomembr. 1415, 147 (1998).

[34] S. Shrivastava and M. F. Schneider, Evidence for twodimensional solitary sound waves in a lipid controlled interface and its implications for biological signalling, J. R. Soc. Interface 11, 20140098 (2014).

[35] T. J. McIntosh and S. A. Simon, Contributions of hydration and steric (entropic) pressures to the interactions between phosphatidylcholine bilayers: Experiments with the subgel phase, Biochemistry 32, 8374 (1993).

[36] E. Schneck, F. Sedlmeier, and R. R. Netz, Hydration repulsion between biomembranes results from an interplay of dehydration and depolarization, Proc. Natl. Acad. Sci. USA 109, 14405 (2012).

[37] J. Holzwarth, Structure and dynamic of phospholipid membranes from nanoseconds to seconds, in The Enzyme Catalysis Process: Energetics, Mechanism and Dynamics, edited by A. Cooper, J. Houben, and L. Chien (Springer Science + Business Media, New York, 1989), pp. 383-410.

[38] B. Krasovitski, V. Frenkel, S. Shoham, and E. Kimmel, Intramembrane cavitation as a unifying mechanism for ultrasound-induced bioeffects, Proc. Natl. Acad. Sci. USA 108, 3258 (2011).

[39] R. N. Lewis, B. D. Sykes, and R. N. McElhaney, Thermotropic phase behavior of model membranes composed of phosphatidylcholines containing cis-monounsaturated acyl chain homologues of oleic acid: Differential scanning calorimetric and 31P NMR spectroscopic studies, Biochemistry 27, 880 (1988).

[40] R. P. Rand, Interacting phospholipid bilayers: Measured forces and induced structural changes, Annu. Rev. Biophys. Bioeng. 10, 277 (1981).

[41] G. Cevc and D. Marsh, Hydration of noncharged lipid bilayer membranes. Theory and experiments with phosphatidylethanolamines, Biophys. J. 47, 21 (1985).

[42] P. J. Rossky, Exploring nanoscale hydrophobic hydration, Faraday Discuss. 146, 13 (2010).
[43] M. Kodama, M. Kuwabara, and S. Seki, Successive phase-transition phenomena and phase diagram of the phosphatidylcholine-water system as revealed by differential scanning calorimetry, Biochim. Biochim. Acta, Biomembr. 689, 567 (1982).

[44] K. Ohsaka and E. H. Trinh, Dynamic nucleation of ice induced by a single stable cavitation bubble, Appl. Phys. Lett. 73, 129 (1998).

[45] B. Lindinger, R. Mettin, R. Chow, and W. Lauterborn, Ice Crystallization Induced by Optical Breakdown, Phys. Rev. Lett. 99, 045701 (2007).

[46] R. Chow, R. Mettin, and B. Lindinger, The importance of acoustic cavitation in the sonocrystallisation of ice-high speed observations of a single acoustic bubble, Proc. IEEE - Ultrason. Symp. 2, 1447 (2003).

[47] R. Hickling, Nucleation of freezing by cavity collapse and its relation to cavitation damage, Nature 206, 915 (1965).

[48] M. N. Plooster, Nucleation of freezing in supercooled water by cavitation, Nature 217, 1246 (1968).

[49] G. A. Lyzenga, T. J. Ahrens, W. J. Nellis, and A. C. Mitchell, The temperature of shock-compressed water, J. Chem. Phys. 76, 6282 (1982).

[50] S. W. Kieffer, X. Lu, G. McFarquhar, and K. H. Wohletz, A redetermination of the ice/vapor ratio of Enceladus' plumes: Implications for sublimation and the lack of a liquid water reservoir, Icarus 203, 238 (2009).

[51] R. A. Shaw, A. J. Durant, and Y. Mi, Heterogeneous surface crystallization observed in undercooled water, J. Phys. Chem. B 109, 9865 (2005).

[52] M. Citroni, S. Fanetti, N. Falsini, P. Foggi, and R. Bini, Melting dynamics of ice in the mesoscopic regime, Proc. Natl. Acad. Sci. U. S. A. 114, 5935 (2017).

[53] R. Chow, R. Blindt, R. Chivers, and M. Povey, A study on the primary and secondary nucleation of ice by power ultrasound, Ultrasonics 43, 227 (2005).

[54] M Blander and J. L Katz, Bubble nucleation in liquids, AIChE J. 21, 833 (1975)

[55] L. D. Landau and E. M Lifshitz, Fluctuations of the fundamental thermodynamic quantities, in Statistical Physics, 3rd ed., Part 1 (Butterworth-Heinemann, Burlington, MA, 1980), p. 338.

[56] A. Einstein, Discussion following lecture version of the present state of the problem of specific heat, in The Collected Papers of Albert Einstein (Princeton University Press, Princeton, NJ, 1911), p. 427.

[57] A. Einstein, Theory of the opalescence of homogenous fluids and liquid mixtures near the critical state, Ann. Phys. 338, 1275 (1910).

[58] S. Halstenberg, T. Heimburg, T. Hianik, U. Kaatze, and R. Krivanek, Cholesterol-induced variations in the volume and enthalpy fluctuations of lipid bilayers, Biophys. J. 75, 264 (1998).

[59] D. B. Tatat and F. Dunn, Ultrasound and model membrane systems: analyses and predictions, J. Phys. Chem. 96, 3548 (1992).

[60] S. Shrivastava, Non-linear solitary sound waves in lipid membranes and their possible role in biological signaling Ph.D. thesis, Boston University, 2014, available at https://open.bu. edu/handle/2144/11049. 
[61] L. D. Landau and E. M. Lifshitz, Entropy for nonequilibrium, in Statistical Physics, 3rd ed., Part 1 (Butterworth-Heinemann, Burlington, MA, 1980), pp. 26-27.

[62] T. Heimburg, Linear nonequilibrium thermodynamics of reversible periodic processes and chemical oscillations, Phys. Chem. Chem. Phys. 19, 17331 (2017).
[63] R. Menikoff and B. J. Plohr, The Riemann problem for fluid flow of real materials, Rev. Mod. Phys. 61, 75 (1989).

[64] L. D. Landau and E. M. Lifshitz, Nucleation in phase transitions, in Statistical Physics, 3rd ed., Part 1 (ButterworthHeinemann, Burlington, MA, 1980), p. 534.

[65] http://researchdata.ox.ac.uk/. 